\title{
Correction: weight, socio-demographics, and health behaviour related correlates of academic performance in first year university students
}

Tom Deliens ${ }^{1 *}$, Peter Clarys ${ }^{1}$, Ilse De Bourdeaudhuij ${ }^{2}$ and Benedicte Deforche $e^{1,2}$

\section{Correction}

After publication of this article [1], we noted an error in the values of the "Initial WC" row of Table 1. The correct values are presented here (Table 1).

Table 1 Descriptive statistics of possible influencing factors of GPA in first year university students (\%, Mean \pm SD, $n=101)$, subdivided into students who passed $(n=52)$, failed $(n=22)$ or did not attend all final course exams $(n=27)$

\begin{tabular}{lllll}
\hline Measures & $\begin{array}{l}\text { All } \\
\mathbf{n}=\mathbf{7 4}\end{array}$ & $\begin{array}{l}\text { Passed } \\
\mathbf{n}=\mathbf{5 2}\end{array}$ & $\begin{array}{l}\text { Failed } \\
\mathbf{n}=\mathbf{2 2}\end{array}$ & $\begin{array}{l}\text { Did not attend all } \\
\text { course exams } \mathbf{n}=\mathbf{2 7}\end{array}$ \\
\hline GPA (\%) & $64.3 \pm 9.2$ & $68.3 \pm 6.9$ & $54.7 \pm 6.2$ & $/$
\end{tabular}

Demographics

Gender (\% of females)

$\begin{array}{llll}77.0 & 82.7 & 63.6 & 40.7 \\ 18.0 \pm 0.6 & 17.9 \pm 0.5 & 18.1 \pm 0.8 & 18.3 \pm 0.9 \\ 20.9 & 23.3 & 15.4 & 20.0 \\ 47.3 & 48.1 & 45.5 & 25.9 \\ 68.6 \pm 7.5 & 70.0 \pm 6.9 & 65.0 \pm 8.0 & 63.6 \pm 6.3\end{array}$

GPA in the last year of secondary school (\%)

$68.6 \pm 7.5$

$70.0 \pm 6.9$

$65.0 \pm 8.0$

46.7

Education father (\% diploma higher education)

$\begin{array}{llll}57.2 & 48.3 & 77.0 & 46.7 \\ 69.0 & 70.0 & 66.6 & 60.0 \\ 95.9 & 96.2 & 95.5 & 96.2 \\ 11.0 & 9.8 & 13.6 & 11.5\end{array}$

Dieting status (\% dieters) ${ }^{b}$

$\begin{array}{llll}61.8 \pm 9.3 & 61.0 \pm 8.0 & 63.5 \pm 12.0 & 66.7 \pm 14.0 \\ 21.7 \pm 2.7 & 21.5 \pm 2.5 & 22.0 \pm 3.1 & 22.1 \pm 3.3 \\ 22.5 \pm 7.1 & 22.8 \pm 7.4 & 21.9 \pm 6.7 & 19.2 \pm 6.5 \\ 70.4 \pm 6.4 & 69.6 \pm 5.6 & 72.3 \pm 7.9 & 73.5 \pm 8.0 \\ 0.7 \pm 2.0 & 0.4 \pm 1.9 & 1.6 \pm 1.8 & 1.6 \pm 2.2 \\ 0.3 \pm 0.8 & 0.1 \pm 0.8 & 0.5 \pm 0.7 & 0.4 \pm 0.8 \\ 1.0 \pm 2.4 & 0.7 \pm 2.5 & 1.5 \pm 2.0 & 0.5 \pm 2.8 \\ -0.0 \pm 2.3 & -0.5 \pm 2.3 & 1.0 \pm 2.1 & 1.2 \pm 2.2\end{array}$

\footnotetext{
*Correspondence: Tom.Deliens@vub.ac.be

'Department of Human Biometry and Biomechanics, Vrije Universiteit Brussel, Brussels, Belgium

Full list of author information is available at the end of the article
} 
Table 1 Descriptive statistics of possible influencing factors of GPA in first year university students (\%, Mean \pm SD, $n=101)$, subdivided into students who passed $(n=52)$, failed $(n=22)$ or did not attend all final course exams $(n=27)$ (Continued)

Physical activity ${ }^{d}$

Active transportation (walking and cycling) (min/week)

Sport participation (min/week)

Total physical activity (min/week)

\section{Sedentary behaviour ${ }^{a}$}

TV/DVD watching on weekdays (hours/day)

TV/DVD watching on weekend days (hours/day)

Reading and studying on weekdays (hours/day)

Reading and studying on weekend days (hours/day)

Computer activities on week days (hours/day)

Computer activities on weekend days (hours/day)

Video games on weekdays (hours/day)

Video games on weekend days (hours/day)

\section{Eating habits}

Eating breakfast (\#/week) ${ }^{a}$

Eating lunch $(\# / \text { week })^{a}$

Eating dinner (\#/week) ${ }^{a}$

Eating at home with parents (\#/week) ${ }^{a}$

Eating at student restaurant $(\# / \text { week })^{a}$

Eating at fast food restaurant (\#/week) ${ }^{a}$

Eating at other kind of restaurant (\#/week) ${ }^{a}$

Eating at a friend's place (\#/week) ${ }^{a}$

Fruit consumption $(\# / \text { day })^{\mathrm{b}}$

Vegetable consumption (\#/day) ${ }^{\text {b }}$

Soda consumption $(\# / \text { day })^{\text {b }}$

French fries consumption (\#/week)

Fast food consumption (\#/week) ${ }^{\mathrm{b}}$

\section{Alcohol}

Frequency of alcohol use (\#/week)

Frequency of alcohol consumptions (\# on drinking days) ${ }^{c}$

\section{Sleeping habits ${ }^{c}$}

Hours of sleep on weekdays (hours/day)

Hours of sleep on weekend days (hours/day)

\section{Stress}

$\begin{array}{llll}179.7 \pm 123.5 & 175.7 \pm 119.5 & 189.5 \pm 135.3 & 193.5 \pm 118.4 \\ 146.1 \pm 180.1 & 152.7 \pm 180.4 & 130.7 \pm 182.9 & 117.4 \pm 161.0 \\ 324.8 \pm 211.7 & 326.8 \pm 220.3 & 320.2 \pm 195.4 & 310.8 \pm 222.8\end{array}$

$\begin{array}{llll}1.2 \pm 0.8 & 1.2 \pm 0.8 & 1.1 \pm 0.8 & 1.2 \pm 0.8 \\ 2.1 \pm 1.1 & 2.1 \pm 1.2 & 1.9 \pm 1.0 & 2.2 \pm 1.3 \\ 1.8 \pm 1.1 & 1.8 \pm 1.2 & 1.9 \pm 0.8 & 1.9 \pm 1.1 \\ 2.9 \pm 1.5 & 2.8 \pm 1.6 & 3.1 \pm 1.5 & 2.8 \pm 1.5 \\ 1.7 \pm 1.3 & 1.7 \pm 1.4 & 1.7 \pm 1.2 & 1.9 \pm 1.2 \\ 1.9 \pm 1.2 & 1.8 \pm 1.3 & 2.1 \pm 1.0 & 2.3 \pm 1.5 \\ 0.2 \pm 0.6 & 0.1 \pm 0.4 & 0.4 \pm 0.9 & 0.3 \pm 1.0 \\ 0.4 \pm 1.0 & 0.3 \pm 0.9 & 0.5 \pm 1.2 & 0.6 \pm 1.1\end{array}$

$5.7 \pm 2.2$

5.52 .3

$6.0 \pm 2.0$

$5.8 \pm 2.3$

$6.6 \pm 1.2$

$6.5 \pm 1.3$

$6.6 \pm 1.0$

$6.7 \pm 0.9$

$6.7 \pm 0.9$

$6.7 \pm 0.7$

$6.6 \pm 1.3$

$6.8 \pm 0.5$

$3.8 \pm 2.1$

$3.6 \pm 2.1$

$4.1 \pm 2.1$

$4.6 \pm 2.1$

$1.2 \pm 1.5$

$1.0 \pm 1.1$

$1.8 \pm 2.1$

$1.8 \pm 1.9$

$0.3 \pm 0.4$

$0.3 \pm 0.4$

$0.3 \pm 0.3$

$0.4 \pm 0.3$

$0.3 \pm 0.3$

$0.3 \pm 0.3$

$0.3 \pm 0.3$

$0.4 \pm 0.3$

$0.4 \pm 0.5$

$0.4 \pm 0.4$

$0.4 \pm 0.6$

$0.5 \pm 0.5$

$1.0 \pm 1.0$

$1.0 \pm 1.1$

$0.9 \pm 0.6$

$1.0 \pm 1.1$

$1.2 \pm 0.7$

$1.2 \pm 0.7$

$1.2 \pm 0.6$

$1.3 \pm 1.0$

$0.8 \pm 1.1$

$0.6 \pm 0.9$

$1.2 \pm 1.3$

$1.2 \pm 1.3$

$0.1 \pm 0.1$

$0.1 \pm 0.1$

$0.1 \pm 0.1$

$0.1 \pm 0.1$

$0.7 \pm 0.9$

$0.7 \pm 0.9$

$0.8 \pm 0.9$

$0.9 \pm 0.9$

$\begin{array}{llll}0.8 \pm 1.5 & 0.6 \pm 1.0 & 1.3 \pm 2.4 & 0.8 \pm 1.3 \\ 2.7 \pm 2.0 & 2.6 \pm 1.9 & 2.9 \pm 2.2 & 3.1 \pm 3.0\end{array}$

$\begin{array}{llll}7.8 \pm 1.0 & 7.8 \pm 1.0 & 7.9 \pm 1.1 & 7.6 \pm 0.9\end{array}$

$9.4 \pm 1.2 \quad 9.4 \pm 1.2 \quad 9.3 \pm 1.3 \quad 9.2 \pm 1.2$




\section{Author details}

${ }^{1}$ Department of Human Biometry and Biomechanics, Vrije Universiteit Brussel, Brussels, Belgium. ${ }^{2}$ Department of Movement and Sports Sciences, Ghent University, Ghent, Belgium.

Received: 3 February 2014 Accepted: 3 February 2014

Published: 8 February 2014

\section{Reference}

Deliens T, et al: Weight, socio-demographics, and health behaviour related correlates of academic performance in first year university students. Nutr J 2013, 12:162.

doi:10.1186/1475-2891-13-16

Cite this article as: Deliens et al:: Correction: weight, socio-demographics, and health behaviour related correlates of academic performance in first year university students. Nutrition Journal 2014 13:16.

\section{Submit your next manuscript to BioMed Central and take full advantage of:}

- Convenient online submission

- Thorough peer review

- No space constraints or color figure charges

- Immediate publication on acceptance

- Inclusion in PubMed, CAS, Scopus and Google Scholar

- Research which is freely available for redistribution 